\title{
Déficit vocacional en la era digital
}

\author{
María Pilar Sánchez Hernández \\ mpilarsanher@gmail.com
}

Resumen: Elegir el futuro profesional nunca ha sido tarea fácil para los jóvenes bachilleres. Si a esto le añadimos la inestabilidad del mercado laboral y el cambio de modelo productivo generado por el impacto de las TIC y la globalización, la decisión es mucho más compleja. Lo cierto es que, dentro de unos años, cuando los actuales jóvenes bachilleres inicien su trayectoria laboral en una sociedad tecnológicamente más avanzada, tendrán que elegir profesiones que aún no existen. En parte, esas nuevas profesiones se formarán de la convergencia de importantes áreas de especialización en ciencias y tecnología como la microinformática, la biotecnología y la física cuántica, además del resultado de la transformación digital que está operando transversalmente en otros muchos sectores de actividad como el de la educación, la salud, el transporte, la logística o el financiero.

Este artículo forma parte del estudio empírico de mi tesis doctoral sobre el déficit de talento femenino en las ingenierías tecnológicas. En este texto trato de explicar la relación entre la digitalización de la economía, el empleo y la educación con la elección de estudios universitarios y el papel que están haciendo la Unión Europea, el Gobierno de España y otros organismos y asociaciones para motivar a las mujeres a estudiar ingenierías tecnológicas o trabajar en el sector TIC.

Palabras clave: sociedad digital, empleo, educación, vocación, jóvenes, género

Abstract: Choosing the right career has never been an easy task for young high school graduates. If we add to this doubt the instability of the labour market, the productive model changed by the impact of the ITC and globalization, the final decision must be more complex. The truth is that, in a short time, when current students start working in a digital society, they will have to choose careers that do not exist yet. In part, the new careers will be formed by the convergence of three important specialization areas: microcomputation, biotechnology and quantum physics, as well as the result of the digital transformation that is being carried out transversally in many other sectors of activity such as education, health, transportation, logistics, business, etc. 
This article is an extract from an empirical study which I am developing in my doctoral thesis on the lack of female talent in the ICT engineering. In this text, I try to explain the relationship among the digitalization of the economy, the employment and education, and the selection of university studies and the role that the Spanish Government, the EU and other institutions and academic associations are playing in this area in order to encourage women to study ICT engineering or even to work in the ICT sector.

Key Words: digital society, employee, education, vocation, young people, gender 


\section{INTRODUCCIÓN}

La sociedad digital en construcción plantea muchas cuestiones y alguna que otra paradoja; aún hoy es pronto para afirmar si las TIC determinarán el futuro de la sociedad o si serán solo un instrumento de control de esta por ciertas clases dominantes. Lo cierto es que la teoría sociológica no soporta sino de forma fragmentada el análisis de este proceso de cambio (Aguinaga, 2016:9).

Este artículo muestra una línea de investigación de la tesis en la que llevo trabajando desde hace más de dos años y que trata sobre la identidad de género en la elección de carrera universitaria relacionada con las TIC. Una tesis que nace de mi propia experiencia laboral en el sector tecnológico, donde llevo trabajando más de veinticinco años. Durante todos estos años he sido también observadora participante del rápido proceso de evolución de las empresas del sector TIC. En síntesis, puedo decir que la automatización de los procesos administrativos ha generado múltiples cambios del modelo productivo y organizativo, la deslocalización de trabajadores por efecto de la globalización de la economía, así como la pérdida de miles de puestos de trabajo que, o bien fueron reconvertidos, o bien están siendo sustituidos por nuevas profesiones relacionadas con tecnologías avanzadas como ciberseguridad, análisis de grandes paquetes de datos (big data) o almacenamiento en la nube (cloud).

Mi hipótesis de trabajo está contextualizada en la sociedad tecnológica o digital, donde actualmente las tecnologías de la información y comunicaciones son el instrumento transversal y el motor económico de todos los sectores de actividad. En este nuevo modelo productivo, la inteligencia es más importante que la fuerza física, por lo que uno de los retos del sistema educativo del siglo xxi es incluir — desde los primeros ciclos de educación - el aprendizaje de competencias digitales y habilidades blandas ${ }^{1}$. El alcance de este reto depende en buena parte de la puesta en marcha de políticas públicas inclusivas, que traten de evitar la exclusión social de mujeres y jóvenes fundamentalmente por discriminación de género o escasez de recursos económicos.

Si bien es cierto que la transformación digital va a aportar multitud de beneficios a la sociedad, también debemos ser conscientes de sus desigualdades. Por ejemplo, mientras España mantiene una tasa de desempleo juvenil por encima de la media de los paí-

\footnotetext{
${ }^{1}$ Se refiere al desarrollo de habilidades de autonomía, gestión del tiempo, confianza, autoliderazgo, empatía, coherencia, integridad, saber escuchar, autocontrol, curiosidad, capacidad de reflexión, proactividad, pasión, motivación intrínseca, lógica divergente, humildad, interés por el aprendizaje continuo, capacidad de síntesis y de argumentación...
} 
ses de la UE, la denominada revolución $4.0^{2}$ está creando ya cientos de miles de nuevos puestos de trabajo en el área de programación que los jóvenes desempleados no pueden ocupar por falta de cualificación. Lo más grave es que tampoco va a poder cubrirse esa demanda a medio plazo si no se consigue aumentar la actual cuota de matriculaciones en ingenierías tecnológicas. Estoy tratando de exponer un problema que no ha pasado desapercibido en las agendas digitales de la Unión Europea y del Gobierno de España. Ambos han impulsado el diseño de programas para orientar o motivar a las niñas al estudio de las STEM (ciencias, tecnologías, matemáticas e ingenierías). Estos programas incluyen entre otros objetivos enseñar a las niñas la utilidad o los beneficios que aportan las ingenierías a la sociedad; visibilizar los estereotipos de género arraigados en la sociedad y buscar referentes femeninos en el campo de las ingenierías con los que las niñas se sientan identificadas.

El artículo comprende nueve apartados incluyendo esta introducción. En el segundo apartado expongo el marco teórico de la sociedad digital y desarrollo el problema social que se plantea en la hipótesis de trabajo. A continuación, en el tercer apartado, describo sucintamente las luces y sombras en el desarrollo de la economía digital en España, que se articula en el cuarto y quinto apartados con la transformación del empleo y el déficit de talento digital. Los nuevos perfiles profesionales y emprendedores digitales son explicados de manera más amplia en el apartado sexto. Seguidamente, en los apartados séptimo y octavo trato el impacto de las TIC en la educación y la falta de vocación en el estudio de ingenierías tecnológicas. Por último, en el apartado de conclusiones esbozo una reflexión sobre el reto que va a suponer a las jóvenes — que constituyen cerca del $50 \%$ de la población española - equipararse con sus compañeros en el trabajo del futuro dominado por las TIC.

Para la elaboración de este artículo he utilizado una metodología mixta. Abarca un análisis cualitativo sobre una extensa revisión bibliográfica y webgrafía, la incorporación de algunos resultados de los grupos de discusión que forman parte del trabajo de campo de la tesis y un análisis cuantitativo basado en datos secundarios de fuentes oficiales como el INE, Ministerio de Educación, Cultura y Deporte y la UE. El resultado de esta investigación me ha servido para redefinir algunos objetivos de la tesis y del trabajo de campo en el que estoy trabajando actualmente.

${ }^{2}$ El concepto de industria 4.0 se refiere a la cuarta revolución industrial que consiste en la introducción de las tecnologías digitales en la industria. 


\section{MARCO TEÓRICO Y CONCEPTUAL}

La sociedad tecnológica o digital se sustenta en un nuevo modelo económico que tiene como materia prima la información y de un uso mucho más cualificado de las tecnologías de la información y de las comunicaciones (TIC). Para empresas como Google o Amazon, los datos son el nuevo petróleo del siglo xxI, ya que los refinan para sacarles el máximo beneficio. En la actualidad, consumidores, empresas y gobiernos debaten sobre el derecho que tienen que tener las personas a decidir sobre la privacidad de sus datos.

La democratización de internet ha generado millones de consumidores en red en todo el mundo. Con tan solo disponer de un móvil, un usuario puede acceder gratis a una amplia gama de entretenimiento audiovisual: música, vídeos y literatura, así como a múltiples aplicaciones para el control y gestión de sus cuentas financieras; o aspirar a llevar una vida más saludable mediante el acceso a programas de dieta y actividades deportivas o incluso la contratación de un coach o entrenador personal que a través de WhatsApp nos monitoriza las veinticuatro horas con la idea de conseguir tener el cuerpo deseado. A medida que avanzamos en la sociedad digital, fabricantes y proveedores de servicios van capitalizando internet, es decir, una cosa es no pagar por el acceso a la red y otra hacerlo por la descarga de contenidos de calidad. Detrás de estos nuevos servicios subyace un trabajo cualificado de búsqueda y de recombinación de conocimiento adaptado a los distintos gustos del consumidor. Las oportunidades de negocio en internet parecen ilimitadas, cada día afloran reinventados servicios de pago.

Las TIC facilitaron tanto la globalización de los mercados financieros como la deslocalización del empleo. El modelo de producción digital permite a las empresas desplazar el capital y la fabricación a países con mejores ventajas fiscales y una regularización laboral laxa. Así, la separación entre el capital, la producción de bienes y servicios y la fuerza laboral provocó un vacío legal que a su vez debilitó los procesos de regulación y control arraigados en la sociedad industrial (Castells, 2007:21). Concretamente en el sector TIC, las consultoras tecnológicas aplicaron el taylorismo digital al desarrollo de software mediante la contratación de mano de obra local barata y poco cualificada, un modelo de negocio muy rentable que a partir del año 2000 y tras la crisis de las puntocom derivó en procesos de externalización y deslocalización de tareas a otros países en busca de trabajadores con salarios más bajos. Estas prácticas no solo han contribuido a la precarización de la ingeniería informática como salida profesional, sino también a la reducción progresiva de matriculaciones. Asimismo, en los últimos años, muchos bachilleres no llegaron a matricularse en estas carreras o incluso las abandonaron en los primeros cursos tras considerar que no merecía la pena sacrificar 
años de estudio para al final llegar a ganar prácticamente lo mismo que un profesional de la hostelería.

Otro factor importante de cambio con relación a la organización del trabajo digital ha sido la introducción del término «flexible» vinculado a la oportunidad que tiene hoy un trabajador de adaptar el trabajo a sus necesidades y a las de su empresa. Este modelo se basa en la ejecución por objetivos, en el que no existe un horario formalmente estipulado ni un lugar de trabajo concreto. El trabajo puede realizarse en cualquier lugar y a cualquier hora, lo que permite conciliar el trabajo profesional con la vida personal siempre y cuando el trabajador cumpla todos los objetivos marcados por la empresa, además de las horas que estos trabajadores tienen que descontar de su tiempo de ocio para formarse continuamente en las nuevas tecnologías. Estos trabajadores reciben de sus empleadores distintos dispositivos móviles: ordenadores portátiles, teléfonos inteligentes, tabletas o incluso el pago de la conexión a internet en sus hogares en caso de que trabajen ciertos días u horas desde sus casas. Visto así dentro de este modelo esclavizador la teoría de la sociedad del ocio es una falacia más.

Actualmente nos encontramos ante la tercera gran transformación global de la historia de la humanidad. En esta fase, se da la convergencia de la microinformática, la biotecnología y la física cuántica, unas áreas de especialización que traerán nuevas fuentes de energía, nuevos materiales y nuevas herramientas y que a su vez van a revolucionar las formas de vivir, de trabajar y de relacionarnos (Tezanos, 2009a:49). Existen dos visiones antagónicas sobre los efectos que este progreso científico puede desencadenar: por un lado, hay teóricos que comparten una visión optimista de estos cambios y vaticinan una sociedad del ocio, donde tendremos acceso a un conocimiento ilimitado, tiempo libre y una mejor salud gracias a los avances en biomedicina e ingeniería genética, mientras que, por otro lado, hay teóricos que tienen una visión más catastrofista de la sociedad del futuro y que defienden que la revolución informática puede arrojar a la indigencia a millones de personas en este planeta, llegando incluso al riesgo de convertirse en una nación de ricos y pobres de la información (Tezanos, 2009b:70-72).

Como también defiende Castaño (2009) en su teoría sobre la brecha digital, tendemos a pensar que las innovaciones tecnológicas llegan primero a las naciones y ciudadanos más ricos para luego, tras un proceso de democratización, llegar a la mayoría, eliminando las diferencias económicas y sociales; al menos esto es lo que ocurrió con la televisión, los teléfonos móviles y otros dispositivos más sencillos de utilizar. La evidencia muestra que esto no siempre ha sido así, es decir, ni todos los países ni todos los ciudadanos se han incorporado al mismo ritmo, y tampoco todos los países disponen de esas tecnologías. Siguiendo esta línea de pensamiento, cabe considerar que ni todas las empresas ni todos los individuos, por el simple hecho de tener acceso a internet, son 
usuarios avanzados. Como concluye Castaño, en esta transformación digital habrá ganadores y perdedores, es decir, los consumidores eligen y se benefician de las empresas que primero adoptan las innovaciones, mientras otras perderán cuota de mercado por no tener esas innovaciones, destruyendo a su vez puestos de trabajo. Unas pérdidas que no afectarán al empleo global, pero sí a nivel micro, es decir, las personas que pierdan su empleo y no tengan los recursos o capacidades que demanda el mercado laboral tendrán mayores dificultades para encontrar un empleo remunerado.

Uno de los objetivos de mi investigación es entender por qué existe una dualidad tan marcada en la elección de estudios de ingenierías entre hombres y mujeres, y también me pregunto que, en el supuesto de que los programas de atracción de niñas al estudio de las STEM (ciencias, tecnologías, matemáticas e ingenierías) cumplan las expectativas esperadas, sería interesante conocer qué funciones se espera que desempeñen las mujeres en el nuevo modelo productivo digital: ¿arquitectas o diseñadoras de software?, ¿programadoras? En este sentido, la cantidad de mujeres en el modelo productivo tecnológico las conducirá a aceptar los trabajos precarizados o peor pagados. Por ello, pienso que la persistencia de los roles de género adquiridos en la división de trabajo industrial nos hará repetir los errores de nuestro pasado reciente. Me refiero a que, durante la época del taylorismo, las mujeres que se incorporaron al trabajo remunerado empezaron ocupando puestos de mecanógrafas o secretarias, mientras los hombres que llevaban años de ventaja laboral copaban los puestos de responsabilidad o jefaturas. El hecho es que, actualmente, el número de mujeres matriculadas en ingenierías tecnológicas no supera el $13 \%$, y esto ya es por sí mismo una desventaja competitiva a la hora de ocupar puestos de responsabilidad en el nuevo modelo productivo digital.

Las diferencias entre sexos y las desigualdades de género han sido ampliamente estudiadas por la sociología y explicadas en un abundante marco teórico. Unas teorías sociológicas que pueden sintetizarse en tres grandes enfoques: en primer lugar, tenemos las teorías que defienden la existencia de una base biológica y que explican las diferentes conductas entre hombres y mujeres. En segundo lugar, las teorías que priorizan la socialización y el aprendizaje de los roles de género.

El enfoque funcionalista defiende el aprendizaje de roles desde la perspectiva del funcionamiento social (Parsons, 1982:77), que básicamente radica en entender los mecanismos primarios que salvaguardan las funciones esenciales del sistema social. La primera asignación sobre un individuo dado en todas las sociedades conocidas es adscriptiva relativa al nacimiento y se produce por la determinación de un sexo, es decir, se nace niño/a en una posición particular y en una unidad familiar específica que presumiblemente no pueden ser cambiados. 
Por otro lado, las teorías sobre la construcción social del género y el sexo aúna teóricos que rechazan las diferencias de sexo como algo determinado biológicamente y el género como algo que se aprende culturalmente. Estas teorías permiten comprender cómo las identidades de género y las diferencias sexuales pueden considerarse como productos construidos socialmente.

Entre los teóricos más destacados de este enfoque se encuentra R. W. Connell, quien explica el proceso de socialización con el que se construye la identidad de género desde distintas perspectivas; para este artículo me interesa destacar la teoría de la socialización que trata de la formación de la identidad de género a través de la adquisición e interiorización de las normas sociales (Connell, 1987:190). Los niños, durante su infancia y hasta que se hacen mayores, reciben de la sociedad una serie de prescripciones, modelos de comportamiento apropiados al sexo de cada uno, al mismo tiempo que los principales agentes responsables de la socialización - la familia, los medios de comunicación, sus grupos de pares y la escuela - hacen que esas expectativas y modelos se concreten en sus entornos, con lo que se construyen las bases apropiadas para los niños. Según este autor, el resultado es una identidad de género que generalmente corresponde a las expectativas sociales asignadas a ese sexo.

Finalmente, Bourdieu enfatiza que las estructuras de dominación masculina han sido producto de un trabajo continuado de reproducción llevado a cabo por agentes singulares, entre los que están los hombres. Estos han ejercido la violencia física y la violencia simbólica desde las instituciones de la familia, la Iglesia, la escuela y el Estado (Bourdieu, 2000:70). La familia es la que asume el papel principal tanto en la reproducción de la dominación como en la visión masculina. La Iglesia, por su parte, cuenta con un clero antifeminista dispuesto a condenar todas las faltas femeninas a la decencia, especialmente en materia de indumentaria, además de inculcar el dogma de la inferioridad natural de las mujeres. Por último, la escuela sigue transmitiendo los presupuestos de la representación patriarcal, una cultura «docta» vehiculada por esta institución a través de sus variantes literarias, filosóficas, médicas o jurídicas que no ha dejado de transmitir un pensamiento y unos modelos arcaicos y un discurso oficial sobre el segundo sexo en el que colaboran teólogos, legisladores, médicos y moralistas y que tiende a restringir la autonomía de la esposa en materia de trabajo.

Bourdieu afirma que el concepto de violencia simbólica implica pensar en el fenómeno de la dominación masculina en las relaciones sociales. Bourdieu utiliza el concepto de habitus para explicar una especie de sentido práctico de lo que hay que hacer en sentido práctico. En el mejor de los casos, los sujetos son razonables más que racionales. Estos dos conceptos sirven para entender el derecho universal que reconoce a los hombres como merecedores de un mejor reparto en la división sexual 
del trabajo de producción y reproducción biológico en detrimento del derecho de las mujeres de obtener una parte equitativa en esa división. Este tipo de violencia, a diferencia de la física, se ejerce de forma suave y a menudo invisible. Según Bourdieu, los actos de conocimiento y reconocimiento entre los dominadores y los dominados llevan a estos últimos — muchas veces sin saberlo - a su propia dominación al aceptar tácitamente los límites impuestos. Estos actos unas veces se reflejan en emociones corporales como vergüenza, humillación, timidez, ansiedad, culpabilidad, y otras se muestran en forma de pasiones y sentimientos como el amor, la admiración o el respeto. Otras veces las manifestaciones son más visibles, como el rubor, la confusión verbal, la torpeza, el temblor, la ira o rabia impotente. En definitiva, todas ellas son maneras de someterse a la opinión dominante, aunque no se haga de buen grado. La teoría sociológica de Bourdieu visibiliza la masculinidad de la estructura patriarcal a través de una explicación de la división asexuada del trabajo de hombres y mujeres, donde deja entrever que las disposiciones femeninas son inculcadas a través de la familia y por todo el orden social (Bourdieu, 2000:70). La mayor diferenciación por género se da en las escuelas de formación profesional, donde podemos ver que las chicas siguen copando los cursos de secretariado, auxiliar de enfermería, administrativo, marketing y comunicación y, en cambio, las especialidades de mecánica, electricidad, electrónica e informática quedan reservadas para los chicos. El incremento progresivo de tituladas universitarias, así como la incorporación masiva de mujeres en nuestras sociedades fueron impulsados por el movimiento feminista. Sin embargo, este resultado no se ha traducido en una representación más equitativa en campos como el científico. En este sentido Bourdieu también investigó y clasificó las especialidades universitarias destinadas o reservadas a cada sexo (Bourdieu, 2000:110).

\section{LUCES Y SOMBRAS EN LA NUEVA ECONOMÍA DIGITAL}

El continuo incremento de internautas en la red está generando un flujo permanente e instantáneo de información que puede almacenarse para darle una utilidad posterior o analizarse con determinadas aplicaciones TIC para transformarla en conocimiento, es decir, en los datos necesarios para crear un sinfín de nuevos negocios. En este contexto, la CEOE estima que el impacto de la digitalización en la economía para 2025 será de unos 120000 millones de euros, equivalente al $11 \%$ del PIB actual.

La digitalización se está implantando a distintas velocidades en los países de la UE y a medida que avanza demanda más talento digital. En la actualidad, un $44 \%$ de los 
europeos carecen de competencias digitales básicas ${ }^{3}$. Por ello, y para estimular la innovación y el empleo digital en los países miembros, se puso en marcha la Agenda Digital Europea (ADE), que estima la creación de alrededor de unos 900000 empleos digitales hasta 2020, que muy probablemente no podrán cubrirse por falta de personal cualifica$\mathrm{do}^{4}$.

Podría afirmarse que España está muy bien posicionada a nivel internacional en materia de digitalización. Por lo general, los índices consultados miden desde el número de dispositivos móviles hasta el perfil de internautas por países o el volumen de compras en línea. En este contexto, España aparece en el undécimo puesto de los países más desarrollados y está en una posición por encima de la media de treinta países analizados en la UE. Sin embargo, los estudios que incluyen indicadores en competencias digitales estiman que solo un $20 \%$ de las empresas españolas ${ }^{5}$ están preparadas para la transformación digital. Una de las principales causas de este retraso señala la falta de competencias digitales en el uso de las nuevas herramientas de gestión como el almacenamiento en la nube o la comunicación a través de redes sociales profesionales. El principal problema es que cerca de un $30 \%$ de los directivos españoles no han recibido formación de manera periódica en competencias digitales, por lo que ignoran cómo planificar la transformación digital en sus empresas. Una cifra que se incrementa en las empresas del IBEX donde encontramos que el $80 \%$ de sus consejeros carece de la experiencia necesaria para conducir a sus organizaciones en la era digital, y lo peor es que solo un $38 \%$ de ellos contempla la estrategia digital como una prioridad (Mckinsey, 2016).

Otros expertos advierten que cuanto más se retrase la transformación digital de las empresas, más se exponen a quedarse fuera del mercado. La mayoría de estos estudios del sector afirman que estas cifras descenderán con el relevo generacional de directivos, y mientras esto sucede, los actuales empresarios optan por la vía rápida, es decir, se alían con otras grandes empresas y organizaciones en búsqueda de grandes ideas o de una aplicación informática útil y rentable que la empresa pueda comercializar rápida-

${ }^{3}$ Datos extraídos del Índice de Economía Digital y Sociedad (DESI), elaborado por la CE y que abarca una serie de indicadores clave sobre la evolución y mejoras de la transformación digital de los países miembros de la UE.

${ }^{4}$ La Agenda Digital Europea, una iniciativa europea que incluye siete objetivos de crecimiento para 2020. Tiene como principal objetivo impulsar el empleo en el sector TIC y fortalecer la innovación en las nuevas industrias.

5 Datos extraídos de un informe del Instituto de Economía Digital realizado en colaboración con Millward Brown: 440 entrevistas — sobre 39 competencias digitales — a empresas de ámbito nacional, por ramas de actividad y mayor volumen de facturación. 
mente en el mercado. También ayudan a los jóvenes estudiantes y emprendedores digitales con becas y subvenciones a fondo perdido para que desarrollen proyectos innovadores dentro de las denominadas incubadoras o viveros empresariales, o en laboratorios de reconocidas instituciones académicas.

\section{LA TRANSFORMACIÓN DEL EMPLEO EN LA ERA DIGITAL}

Prácticamente todos los días, los medios se hacen eco de los efectos que la llamada revolución industrial 4.0 va a tener en el mercado laboral. Los cambios tecnológicos siempre han ido acompañados de destrucción de empleo, por ello, uno de los temas recurrentes en la prensa es la automatización o robotización de los puestos de trabajo. Es comprensible que en el imaginario colectivo se mantenga la idea de la pérdida de empleo cuando el debate está presente en todos los sectores económicos. Así, en el último encuentro del Foro Económico Mundial de Davos se estimó que, para 2020, como consecuencia de los cambios tecnológicos y demográficos, se perderán hasta siete millones de puestos de trabajo, de los cuales dos tercios estarán relacionados con rutinarios trabajos de oficina o funciones administrativas. En el Foro también se habló de la creación de nuevas profesiones en campos como la energía, el medioambiente, la inteligencia artificial, bioinformática, biología sintética, etc.

En este sentido, la economía digital en Francia está generando ya en torno al 25\% del nuevo empleo, mientras en España el sector TIC en 2015 empleó un 6,1\% más que el año anterior. Un crecimiento anual progresivo que sitúa al sector TIC como el que más empleo genera después del sector turístico (Informe Anual del sector TIC y de los Contenidos, 2016).

De momento, y como se puede apreciar en el gráfico 1, la distribución del empleo en España por ramas de actividad y sexo mantiene un patrón de comportamiento marcado por los tradicionales roles de género. Los datos muestran un porcentaje mayor de hombres que trabajan en los sectores de transporte, construcción, manufactura y agricultura. Mientras que los porcentajes de mujeres son significativamente mayores en los trabajos que guardan relación directa con los cuidados en los sectores de sanidad, educación, comercio y empleo doméstico (Gráfico 1).

La transformación digital va ligada a la innovación; en este punto, en una encuesta reciente realizada por Sigma Dos, se preguntaba a una muestra representativa del conjunto de españoles su opinión sobre la digitalización de los puestos de trabajo. Los resultados mostraron que aproximadamente un $66 \%$ de estos considera que la automatización de su trabajo no ocurrirá antes de quince años, mientras otro $52 \%$ de los encues- 


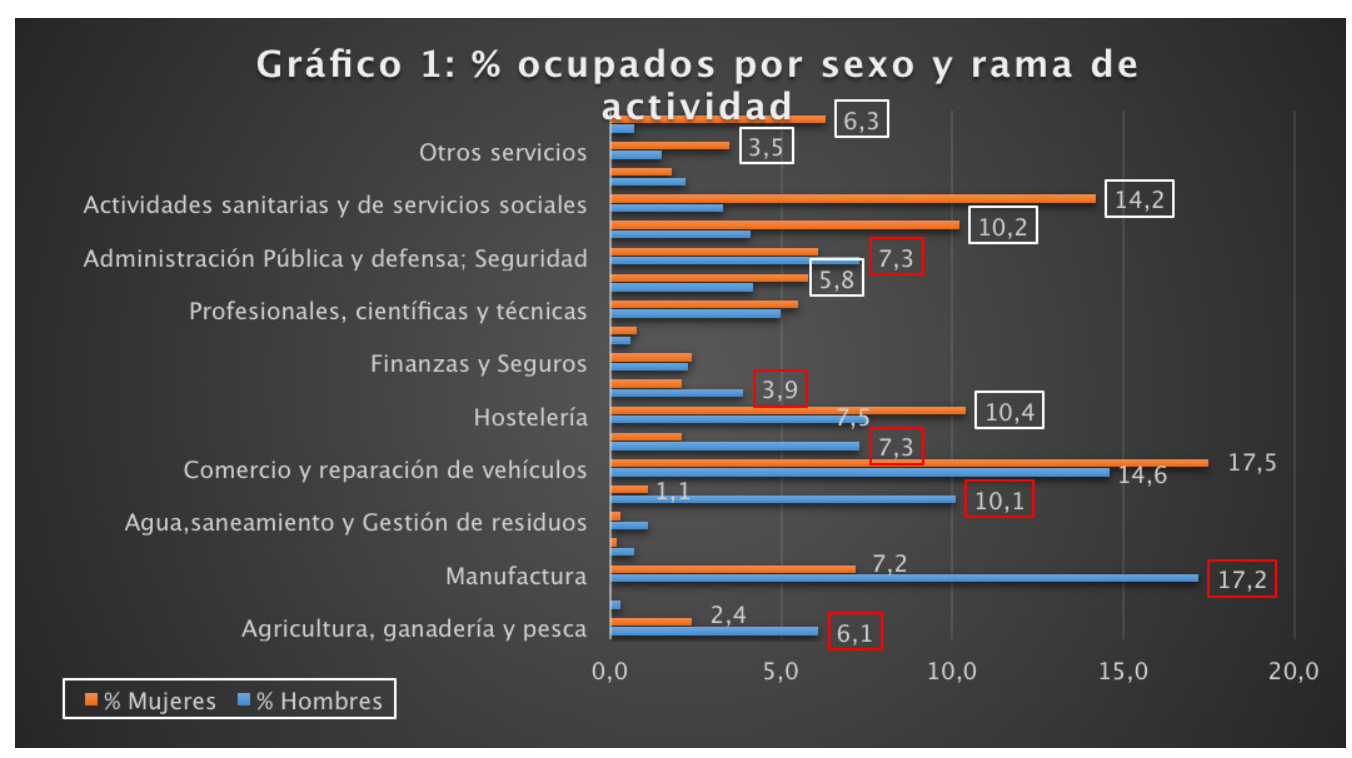

Fuente: Elaboración propia datos sobre datos EPA 2017T1 INE

tados sí cree que sus empleos están en peligro de extinción y que se crearán otros nuevos puestos de trabajo. Lo interesante de este estudio es la valoración que hacen los encuestados sobre las nuevas competencias digitales y el nivel educativo como un factor determinante tanto para la confianza en el presente cambio tecnológico como en la capacidad individual para competir en el nuevo mercado de trabajo. Los datos del estudio reflejan que un tercio de la población carece de capacidades digitales, de los cuales un $51 \%$ no se siente capaz de adaptarse y, aún peor, un $40 \%$, aunque quisiera intentarlo, no cuenta con recursos económicos para reciclarse. Paradójicamente, un $60 \%$ de los que no tienen recursos pertenecen al colectivo de jóvenes con más dificultades para encontrar empleo, siendo a su vez los más convencidos de que el cambio tecnológico lo generará ${ }^{\text {. }}$

Por el momento, el empleo en el sector TIC presenta una distribución regional de las empresas muy concreta. En este sentido, las comunidades autónomas de Madrid y Cataluña acaparan el 55\% del total de las empresas y el 95,9\% del empleo. El tipo de servicios predominante se centra en la edición de programas informáticos, programación, gestión de recursos informáticos, consultoría, procesos de datos, almacenamiento y servicios de reparación y mantenimiento (Informe Anual del Sector TIC, 2016:35), aunque el hecho de que las empresas tecnológicas no estén presentes en una comunidad

${ }^{6}$ Según un estudio publicado recientemente por la Fundación COTEC y Sigma Dos sobre la percepción social de la innovación en la sociedad española. 
autónoma no implica que no pueda contratar personal. Como ya he comentado en otros apartados del artículo, las funciones de programación o edición de programas informáticos no requieren la presencia del trabajador en la empresa, ya que pueden realizarse desde cualquier lugar y a cualquier hora.

\section{DÉFICIT DE TALENTO DIGITAL EN EL SECTOR TIC}

El déficit de talento digital en el sector TIC fue uno de los temas de debate en el último encuentro de economía digital organizado por AMETIC (Asociación Española de Empresas TIC). Entre las conclusiones, destacaron que hay dos factores que están frenando la falta de talento: el primero es la escasa remuneración de los profesionales tecnológicos españoles frente a otras potencias, y el segundo es el escaso interés de los jóvenes en cambiar de residencia por motivos laborales. Desde mi punto de vista, los factores aducidos en el encuentro de AMETIC sobre el déficit de talento digital en España son un tanto sesgados, ya que lo cierto es que existe una significativa brecha salarial entre países y una feroz competencia entre empresas tecnológicas por atraer y contratar jóvenes talentos, además de la congelación y reducción de salarios como acicates de la precarización del trabajo de los ingenieros en el mundo en general y en España en particular. En este sentido, en Silicon Valley - la meca de la tecnología - la cultura del esfuerzo, el sacrificio y las capacidades o habilidades profesionales ya no son garantía de éxito. En un mundo globalizado estos hechos llegan a todos los rincones del planeta y generan desmotivación tanto en la matriculación de estas ingenierías como en el ingrato y mal pagado trabajo en este sector.

Tampoco creo que pueda tratarse a la ligera el cambio de residencia por motivos laborales señalado en el estudio de AMETIC como factor de riesgo en el déficit de talento digital; lo que quiero decir es que no se puede esperar que los jóvenes españoles acepten la movilidad laboral sin un salario que permita su emancipación. En este sentido, hay muchos trabajos e investigaciones que explican los procesos de socialización, la capacitación juvenil y la compresión del riesgo de la emancipación juvenil (Comas, 2004).

En cualquier caso, España es un país con un sector TIC muy masculinizado, y mientras no haya más referentes femeninos seguirá habiendo déficit de talento digital. Como se puede observar en el gráfico 2: «Profesionales e investigadores del sector TIC por sectores y género», de cada cien profesionales trabajando en el sector TIC hay 77 hombres frente a 23 mujeres. Una desigualdad igualmente significativa en el gráfico 3: «Investigadores del sector TIC por género», en el que puede observarse que de cada 100 investigadores solo 31 son mujeres. 


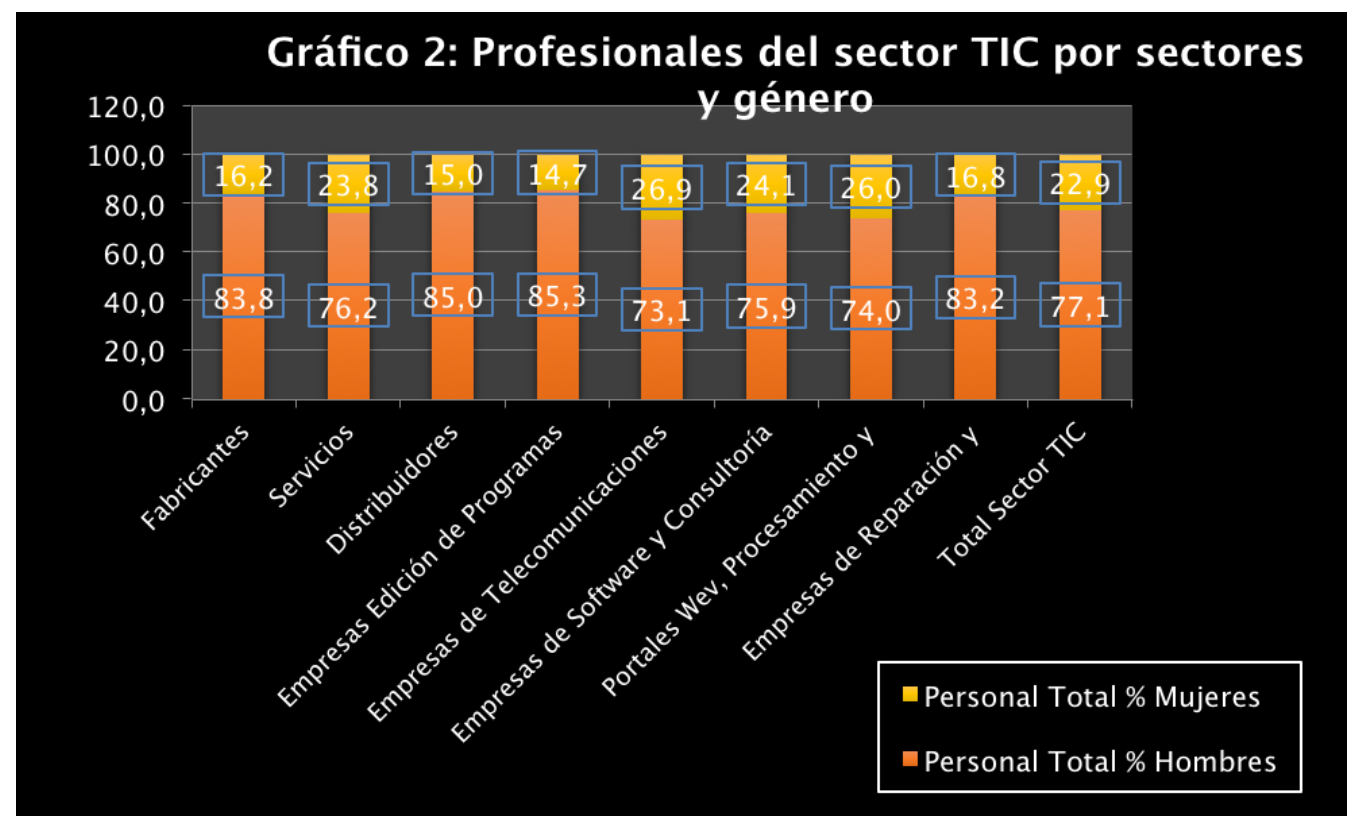

Fuente: Elaboración propia de datos sobre actividades de investigación científica y desarrollo tecnológico (INE, 2015)

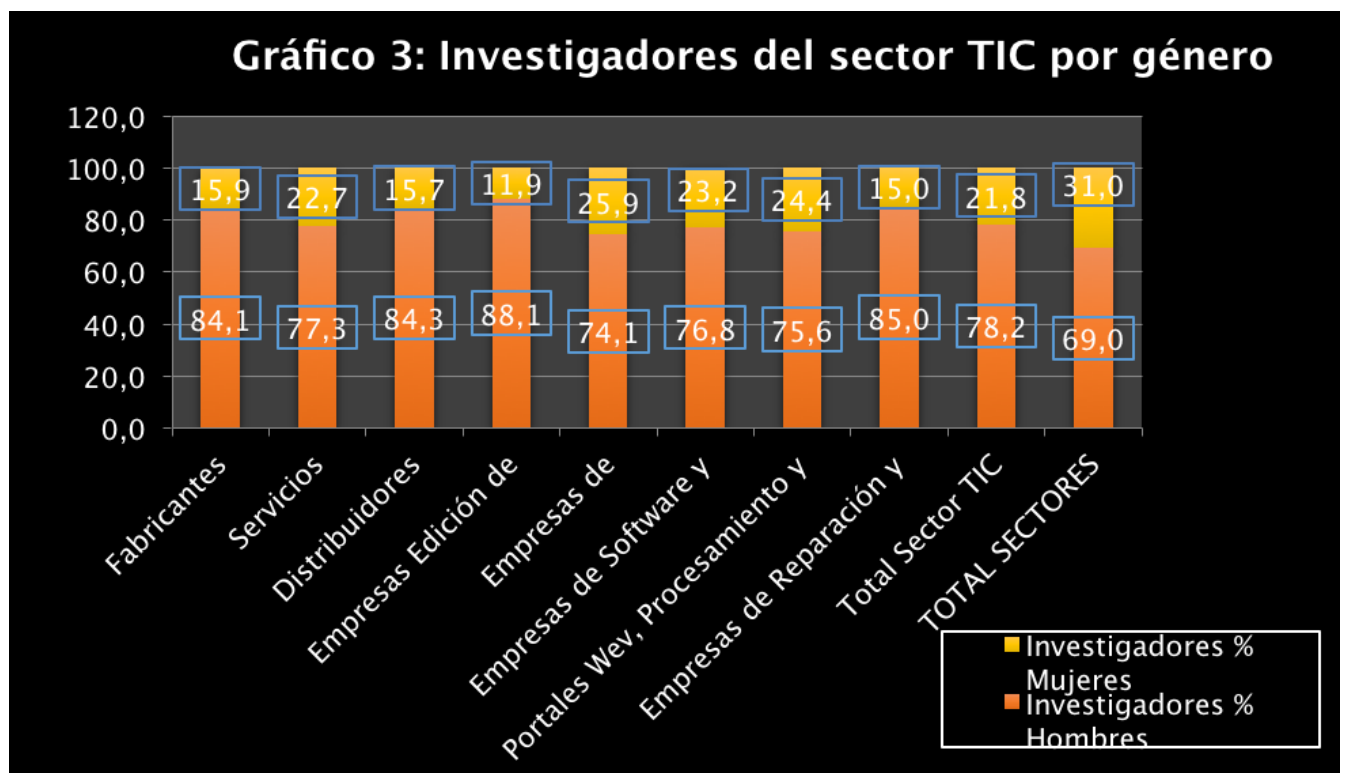

Fuente: Elaboración propia de datos sobre actividades de investigación científica y desarrollo tecnológico (INE, 2015) 
La falta de vocación en el sector TIC no es un problema exclusivo de España; de igual modo, en Europa el $84 \%$ de los profesionales en nuevas tecnologías son varones. Para cumplir con los objetivos de las distintas agendas digitales, la ITU puso en marcha la iniciativa Día Internacional de las Niñas en las TIC con el respaldo de los Estados miembros. El objetivo de esta iniciativa es crear un entorno mundial que empodere a las niñas y mujeres jóvenes y las estimule a considerar el campo de las TIC como carrera profesional. En la misma línea de actuación, el Foro Económico Mundial afirma: «Si la brecha de género persiste en la industria TIC y la transformación del mercado laboral hacia nuevos y emergentes roles relacionados con la informática, la tecnología y las ingenierías no consigue aumentar la tasa de mujeres que actualmente trabajan en estos oficios, el colectivo de mujeres corre el riesgo de salir perdiendo el día de mañana en lo que a oportunidades laborales se refiere», (World Economic Forum, 2016). Actualmente solo hay un $4 \%$ de consejeras delegadas y cerca de un $30 \%$ de mujeres en las empresas dedicadas a las STEM, según datos de las asociaciones Universia ${ }^{7}$ y Womenalia ${ }^{8}$, quienes, además, estiman que para el año 2025 el 50\% de las profesiones estarán relacionadas con las nuevas tecnologías.

Asimismo, hay otras asociaciones académicas en el campo de las STEM y organizaciones sindicales preocupadas por la falta de talento femenino en el sector TIC. Sin ir más lejos, la Asociación de Mujeres Investigadoras y Tecnólogas (AMIT) considera que es necesario el apoyo de políticas adecuadas para evitar que la situación empeore. Sus apreciaciones se basan en el hecho del descenso de matriculaciones de mujeres en carreras STEM y ponen el ejemplo de Zaragoza, donde se ha reducido el número de matriculaciones de un $25 \%$ en 1998 a un $9 \%$ en 2016. En esta misma línea, un estudio reciente de la UGT coincide en señalar que el sector digital dejará de contemplarse como tal, ya que hay una clara tendencia a la digitalización de todos los sectores. En este contexto, UGT también afirma que la escasa presencia de trabajadoras en el sector TIC conllevará un deterioro de la ya difícil situación laboral de las mujeres en el conjunto del mercado de trabajo (Mujer y Tecnología, 2016:6).

La sociología también ha investigado el tema de la brecha digital de género. Uno de sus mayores exponentes es la socióloga Cecilia Castaño - experta en el estudio de género, ciencia y tecnología-, quien ha cuestionado por qué no hay más mujeres estu-

${ }^{7}$ La Fundación Universia dispone de una red de cooperación universitaria de habla hispana y portuguesa constituida por 1401 instituciones de educación superior en 23 países de Iberoamérica, con el objetivo de impulsar la inclusión laboral de las personas con discapacidad.

${ }^{8}$ Womenalia es una red de mujeres profesionales que tiene como objeto mejorar el empleo, la carrera profesional o impulsar el emprendimiento de este colectivo. 
diando o trabajando en el sector de las tecnologías de la información, cuando actualmente tenemos más mujeres que hombres con estudios universitarios (Castaño, 2010a). Castaño considera que la brecha digital de género proviene de un problema de actitudes y estereotipos sociales. La falta de mujeres en un sector en auge como es el de las TIC es una rémora para la competitividad y una concepción excesivamente masculina y tecnológica de la innovación. Es necesario definir una estrategia desde la perspectiva de la diversidad que incluya a las mujeres en las actividades de emprendimiento, creatividad e innovación. El hecho de que los hombres hayan llegado antes a las TIC ha contribuido a que los programas se hayan diseñado a la medida de sus necesidades y no a las de las mujeres (Castaño, 2010b).

El estudio de las condiciones laborales refleja que la contratación temporal unida al tiempo parcial es un 52,9\% mayor en las mujeres que en los hombres, a pesar de que su nivel educativo ha aumentado en mayor proporción que el de los hombres. Los salarios son de media también más bajos, lo que refleja la tremenda desigualdad entre ambos sexos en el mundo laboral. Esta situación ha dejado a las mujeres en una situación tan vulnerable que facilita el que se conviertan en trabajadores ideales de la economía capitalista global en red. La necesidad obliga y, en ese sector, ellas pueden trabajar eficientemente y adaptarse a los requerimientos cambiantes de las empresas, aunque tanto su remuneración como sus posibilidades de promoción sean menores que las de sus compañeros (Castells, 2009:58).

\section{NUEVOS PERFILES PROFESIONALES Y EMPRENDEDORES DIGITALES}

En paralelo a la precarización del empleo TIC, el sector tecnológico no cesa de innovar en nuevas tecnologías y crear nuevas profesiones en áreas como la ciberseguridad, el almacenamiento en la nube o el internet de las cosas. En este sentido, el aumento de la demanda de profesionales especializados en estas áreas genera una guerra entre empresas para atraer el escaso talento, lo que a su vez ha generado una constante subida de salarios. Estos profesionales requieren una formación más especializada que no encuentran fácilmente en el sistema educativo, por lo que se ven obligados a comprar educación privada que requiere un desembolso de capital fuera del alcance de muchos jóvenes. Hay empresas que invierten en este tipo de formación y, para retener el talento, se ven forzadas a cuidar o blindar el contrato de estos profesionales para evitar que sean contratados por la competencia.

Debido a la escasez de profesionales en el sector TIC, las empresas han creado un modelo de organización mixto que contempla un espacio de trabajo y otro de ocio, don- 
de, por supuesto, hay conexión wifi para que los trabajadores continúen su trabajo en la zona de descanso. Estos espacios suelen contar con gimnasio, videojuegos, billar, restaurantes temáticos e incluso un lugar para dejar las mascotas. Estas prácticas tienen un fin «perverso», y es que los empleados se sientan como en las casas que no pueden comprar y permanezcan más tiempo en las oficinas. Podría incluso considerarse como una parte del salario en especie.

La aceptación de los jóvenes de este nuevo estilo de trabajo tiene que ver también con su proceso de socialización. La juventud actual ha sido socializada de una manera muy estricta en el paradigma de «gran futuro» y en un modelo de vida ligado a él (Aguinaga y Comas, 2013a). Sin embargo, tienen que hacer frente a un modelo productivo del que no tienen referentes, es decir, como explican Aguinaga y Comas:

Disponen de herramientas y habilidades que las anteriores generaciones no tuvieron, lo que les permite enfrentarse a dificultades con más capacidad que las anteriores, pero, a la vez, las instrucciones de uso que les venía proporcionando la sociedad (a través de las instituciones, las familias y los adultos) han desaparecido. Esto les obliga a analizar y a elegir, es decir, sin ningún referente previo y en un entorno en el que no es sencillo orientarse, y que se percibe como una maraña en la que no parece fácil determinar prioridades (Aguinaga y Comas, 2013b).

En ese paradigma del gran futuro, no esperaban ganar un salario inferior al que ganaban sus padres. Así, un estudio sobre movilidad social vía ingresos, publicado en la revista Science, permite sacar algunas conclusiones sobre la desigualdad de oportunidades en EE. UU. de las que es posible establecer algunos paralelismos con el entorno europeo, dada la similitud en el modelo productivo. Este estudio revela que un $92 \%$ de los jóvenes americanos nacidos en 1940 — y que en 1970 tenían treinta años — acabaron ganando un salario mayor que el de sus padres. Sin embargo, un 50\% de los nacidos en 1984 obtienen hoy menos ingresos que sus progenitores a su misma edad. Esta reducción salarial ha sido general en la clase media, mientras que en ese mismo período han aumentado los ingresos de los más ricos (Chetty et al., 2016).

La reducción de salario y la pérdida del trabajo estable son dos características del modelo productivo posfordista. En este punto, Bauman cita a Daniel Cohen, un célebre economista de la Sorbona, para ilustrar cómo en pleno apogeo del fordismo los trabajadores estaban unidos con una cadena invisible a su lugar de trabajo, es decir, los trabajadores que iniciaban su carrera profesional en Ford o en Renault tenían la certeza de terminarla en el mismo sitio, mientras un trabajador que empieza hoy su carrera en Microsoft no tiene ni idea de dónde la terminará (Bauman, 2000a). 
La cultura del emprendimiento surgió con las políticas de austeridad aplicadas en la época de Reagan en Estados Unidos y de Thatcher en el Reino Unido, y con el nuevo siglo se ha extendido al resto de Europa. Se trata de un movimiento que ha inspirado a las generaciones nativas digitales (mileniales) para crear microempresas o start ups; jóvenes que sueñan con crear el superproducto digital que les haga tan millonarios como a Bill Gates o Steve Jobs. En los primeros años del movimiento, el analista Leadbeater puso en evidencia la falta de políticas públicas y subvenciones a las empresas impulsoras de la cultura emprendedora, dejando a los emprendedores a su suerte, asumiendo la individualización del riesgo, los costes de sus proyectos y su autofinanciación, además de la responsabilidad total en la gestión, formación, desarrollo profesional, seguros, costes de seguridad social, permisos de maternidad, etc. El autor calculó que un emprendedor empleaba un promedio de veinte horas a la semana para buscar nuevos proyectos y gestionar las incidencias de los anteriores, además de la actualización de los conocimientos necesarios para iniciar el posible nuevo proyecto (Leadbeater, 1999).

Los emprendedores trabajan hoy en sectores en pleno auge dentro de la economía digital como son el diseño, la moda, los productos multimedia y servicios de internet. Una nueva raza de empresarios entre los veinte y los treinta años, que emprenden a través de micronegocios o como autónomos y que a menudo actúan como productores, diseñadores, distribuidores y promotores al mismo tiempo. Sus principales activos son la creatividad, la capacidad de aprendizaje de nuevos conocimientos, la ingenuidad y la imaginación.

La economía digital está generando tipos ideales de emprendedores. Por ejemplo, el nómada del conocimiento es un término acunado por Moravec, vinculado al fenómeno de la «gentrificación del trabajo», que define un perfil de joven profesional y milenial que rechaza el modelo productivo digital, y que huye de trabajos que exigen movilidad y disponibilidad veinticuatro horas. Estos jóvenes dicen renunciar a salarios altos a cambio de hábitos de vida más tranquilos, desempeñando oficios tradicionales como el de barbero o panadero. La diferencia es que estos jóvenes son nativos digitales, han adquirido competencias digitales y tienen la capacidad de adaptar los oficios tradicionales al estilo consumista de la sociedad digital.

En definitiva, la gentrificación del trabajo es una de las oportunidades que ofrece la nueva economía digital, aunque falta matizar que tal oportunidad es desigualitaria, ya que, además de aprender un oficio tradicional y tener competencias digitales, es imprescindible disponer de un capital para iniciar el negocio que conlleva la apertura de un local o el establecimiento de una empresa, y esto no está al alcance de todos los mileniales.

El fenómeno de la gentrificación del trabajo fue estudiado en la Universidad de Princeton centrado en un caso de un barrio de Nueva York. Unos de los mercados urbanos renovados ha sido el de Chelsea, donde, además de una gran variedad de locales y 
restaurantes gastronómicos, pueden encontrarse barberías, panaderías y carnicerías que venden una imagen de producto o servicio artesano, natural, ecológico y sostenible, pero no asequible a todas las personas (Ocejo, 2015).

Hoy podemos encontrar mercados de características similares en España, como el de San Miguel en Madrid, donde se ha conservado la fachada, pero ni los propietarios de los negocios, ni los productos, ni los precios, ni los clientes que lo frecuentan hoy tienen el mismo estatus social que los que lo hacían antes de la reforma. Es precisamente alrededor o dentro de estos mercados renovados donde se está produciendo la gentrificación de los viejos oficios.

\section{LA TRANSFORMACIÓN DIGITAL EN LA EDUCACIÓN}

En el ámbito escolar y, concretamente, en España, hay un ordenador por cada tres alumnos con una conexión a internet del 92,7\% sobre un total de 8108884 alumnos de enseñanzas de régimen general no universitarias ${ }^{9}$. En este punto, cabe señalar que el proceso de integración de las TIC en el aula empezó por dotar a estas de dispositivos electrónicos tales como ordenadores, tabletas o pizarras digitales antes de formar a los profesores en las competencias básicas de uso y aprendizaje. España ha superado como país prácticamente la brecha digital relacionada con el acceso o recursos en la escuela, ahora se trata de superar una nueva brecha relacionada con la alfabetización digital y que ha surgido con el cambio de modelo productivo. Lo cierto es que no sirve de nada estar conectado si no se sabe utilizar los dispositivos o enseñar con ellos.

En este punto, el Parlamento Europeo trata de impulsar la digitalización señalando las ocho competencias clave que todo ciudadano debería adquirir. Tener competencias básicas en el uso de ordenadores para obtener, evaluar, almacenar, producir, presentar e intercambiar información, comunicarse y participar en redes de colaboración a través de internet garantiza un uso seguro y crítico de las tecnologías de la sociedad de la información (TSI) en el trabajo, el ocio y la comunicación (CE, 2006:15).

A estas alturas, es indiscutible que el aprendizaje de estas competencias requiere una metodología educativa que enseñe primero a los profesores a enseñar esas competencias digitales a sus alumnos. En este punto, el sistema educativo prevé un cambio de tendencias a corto, medio y largo plazo con el aumento significativo de asignaturas específicas de programación (coding), además de otras habilidades en el aula como el aprendizaje colaborativo dentro de un espacio de aprendizaje similar al que se está imponiendo en el espacio laboral (Informe Horizon, 2016).

\footnotetext{
9 Datos extraídos del informe de datos y cifras del curso escolar, 2016.
} 
Con la irrupción de las TIC en el entorno laboral, educativo y social, lo prioritario era tener el control de tu ordenador y no dejar que este te controlara a ti; saber lo que un ordenador puede o no puede hacer (Morgan, 1998). Ahora, la alfabetización digital implica desarrollar un conjunto de habilidades sociocognitivas que permitan al alumno seleccionar, procesar y analizar la información. El aprendizaje hoy requiere de la participación y colaboración entre los alumnos, entendido como un proceso progresivo de incorporación como miembro de una comunidad, así como aceptar sus formas de comunicación y actuar en concordancia con sus normas culturales. Las TIC no son una simple herramienta, sino que son el elemento central para la selección, la reconstrucción del conocimiento, la participación y la comunicación.

En este sentido, se han llevado a cabo diversos estudios como el realizado por la Universidad de Valencia ${ }^{10}$, en el que se encontró en general un nivel bajo en la enseñanza de competencia digital, es decir, los profesores dominan los recursos tecnológicos, pero no llegan a tener un uso avanzado de sus funcionalidades. Este estudio concluye que la formación tanto tecnológica como pedagógica generará la autoconfianza necesaria para que el profesor las utilice primero desde el ámbito personal-profesional y después en el aula (Almerich et al. 2010a). Las respuestas del profesorado han dejado constancia de que, a pesar de haber participado en programas de formación, persiste una falta de confianza en la utilización de las TIC en el aula. Alcanzar un óptimo nivel de competencias digitales requiere grandes inversiones de tiempo y dinero: estamos hablando de formar en España a un colectivo de profesorado de régimen general no universitario que alcanza los 682258 profesores (datos y cifras del curso escolar 2015/16). La Administración ha optado por que parte del problema se solucionará con el tiempo. Así las cosas, cabe pensar que el relevo generacional dará paso a una generación de profesores formada en el tercer nivel de competencias relacionadas con las TIC tanto a nivel técnico como pedagógico.

\section{DÉFICIT VOCACIONAL EN EL ESTUDIO DE INGENIERÍAS TIC}

La elección de carrera universitaria no es un tema baladí, a sabiendas de que en cinco años aparecerán en el mercado laboral nuevas profesiones relacionadas con las

${ }^{10}$ El estudio forma parte del proyecto PROFORTIC, cuyo objetivo es detectar las necesidades de formación del profesorado en TIC — en los centros de primaria y de secundaria en la Comunidad Valencianapartir del nivel de competencias en el uso de los recursos tecnológicos. 
TIC. Entre mis objetivos de investigación para la tesis en la que estoy trabajando, trato de conocer en qué carreras se están matriculando los jóvenes, qué aspiraciones tienen en el contexto de un mundo en permanente cambio y cuáles son sus referentes a la hora de elegir sus estudios universitarios.

El gráfico 4 abajo muestra las diferencias entre los jóvenes de ambos sexos en la elección de carrera universitaria. Los datos muestran que entre las dieciocho primeras disciplinas por número de licenciados, el $80 \%$ de matriculación femenina está concentrado en las disciplinas de trabajo social, enfermería y educación, mientras que más del $60 \%$ de matriculaciones masculinas están relacionadas con la actividad física y el deporte, historia o filosofía. A la vista de estos resultados, se confirma que, al igual que en el ámbito laboral, la elección de carrera continua está marcada por la separación entre actividades de producción y reproducción, que pueden explicarse con las teorías de alcance medio expuestas en el marco teórico sobre el aprendizaje de los roles de género basadas en los ensayos de Bourdieu ${ }^{11}$, las teorías funcionalistas cuyo exponente máximo es Parsons ${ }^{12}$ y otros ensayos de Connell sobre la construcción social del género ${ }^{13}$.

Otro factor a tener en cuenta en la elección de carrera universitaria son las aspiraciones de los jóvenes adolescentes españoles. En este sentido, un estudio reciente muestra que un $43 \%$ de los chicos frente a un 17\% de chicas de entre 16 y 19 años prefiere estudiar carreras relacionadas con las áreas de ingeniería, arquitectura o una carrera científica. En cambio, el 79\% de chicas eligen estudiar medicina, derecho y ciencias sociales (Educa 2020, GAD3, 2017a). Estos datos corroboran los resultados de los gráficos 4 y 5 .

El gráfico 5 más abajo muestra los datos de matriculación en carreras técnicas y de ciencias. En la educación superior, las mujeres representan solo el $35 \%$ de matriculaciones en esas disciplinas, y todavía es menor en las disciplinas TIC, ingenierías, fabricación y construcción. Las mujeres abandonan las disciplinas STEM en mayor número durante la educación superior, en su transición al mundo laboral e incluso durante su trayectoria profesional. De veinte carreras que se incluyen en el gráfico, las mujeres destacan tan solo en cinco: ciencias, química, biología y veterinaria, seguida por muy poca diferencia por arquitectura. Cabe destacar el incremento de matriculaciones de

${ }^{11}$ Bourdieu, P. (2000), en la dominación masculina analiza las estructuras de dominación masculina (Iglesia, familia y Estado) y el trabajo de reproducción social que han ejercido sobre las mujeres a través de la violencia simbólica.

12 Parsons, T (1982), establece una analogía entre el sistema social y el organismo humano.

${ }^{13}$ Connell, R. W. (1987) trata la formación de la identidad de género a través de la adquisición e interiorización de normas sociales. 


\section{Gráfico 4: \% de estudiantes Grado, por grupo de edad, sexo y campo de estudio 2015/16}

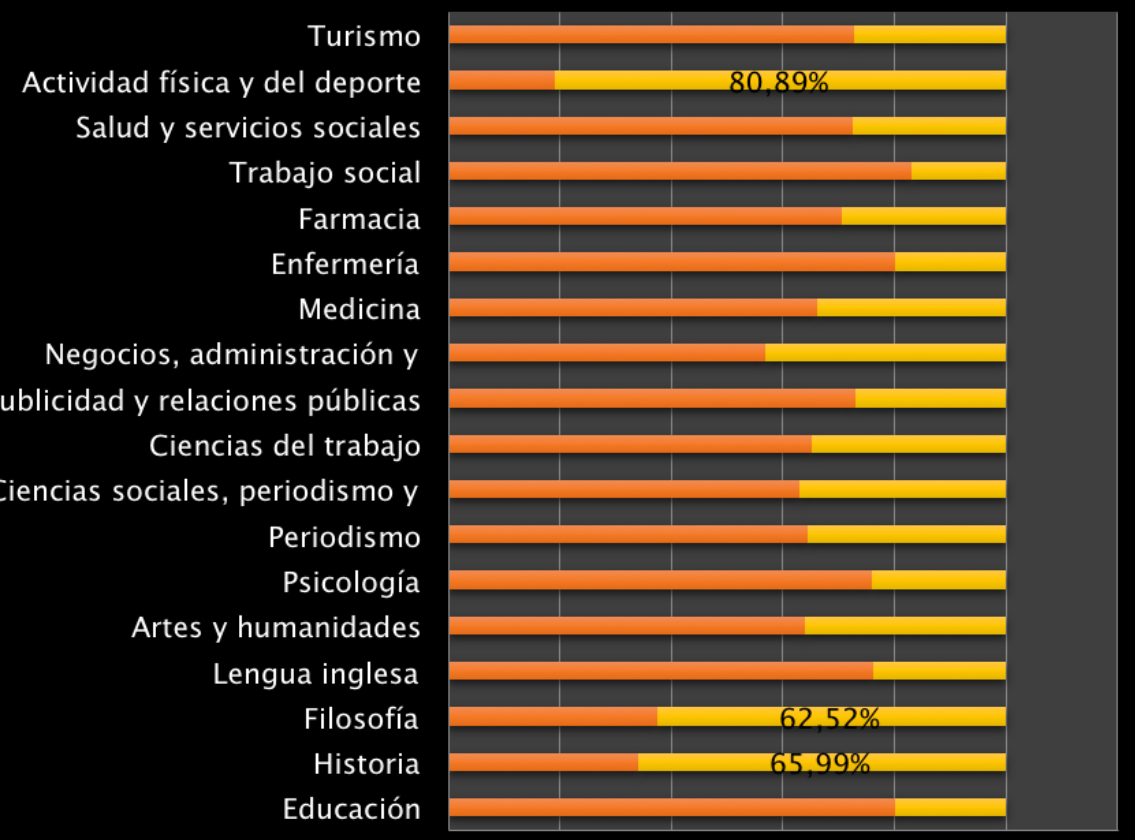

$0,00 \% \quad 20,00 \% 40,00 \% 60,00 \% 80,00 \% 100,00 \% 120,00 \%$

\section{-\% Mujeres $\square \%$ Hombfres}

Fuente: Elaboración propia sobre datos S. G. de coordinación y seguimiento universitario. Ministerio de Educación, Cultura y Deporte

mujeres en dos carreras vinculadas con las nuevas tecnologías: biotecnología y ciencia y tecnología de los alimentos. Como puede apreciarse en este gráfico, en general los hombres dominan la mayoría de ingenierías y, en mayor número, las relacionadas con las TIC.

En mi trabajo de investigación descubrí que uno de los factores de decisión más notoria en la elección de carrera universitaria, en los grupos de discusión de los jóvenes bachilleres, era la renta de la familia. Los jóvenes entendían que estudiar medicina o ingeniería podía garantizar una salida laboral, pero los años de estudio y el coste de la matrícula eran inadmisibles para algunas familias. En cambio, en el grupo de discusión 


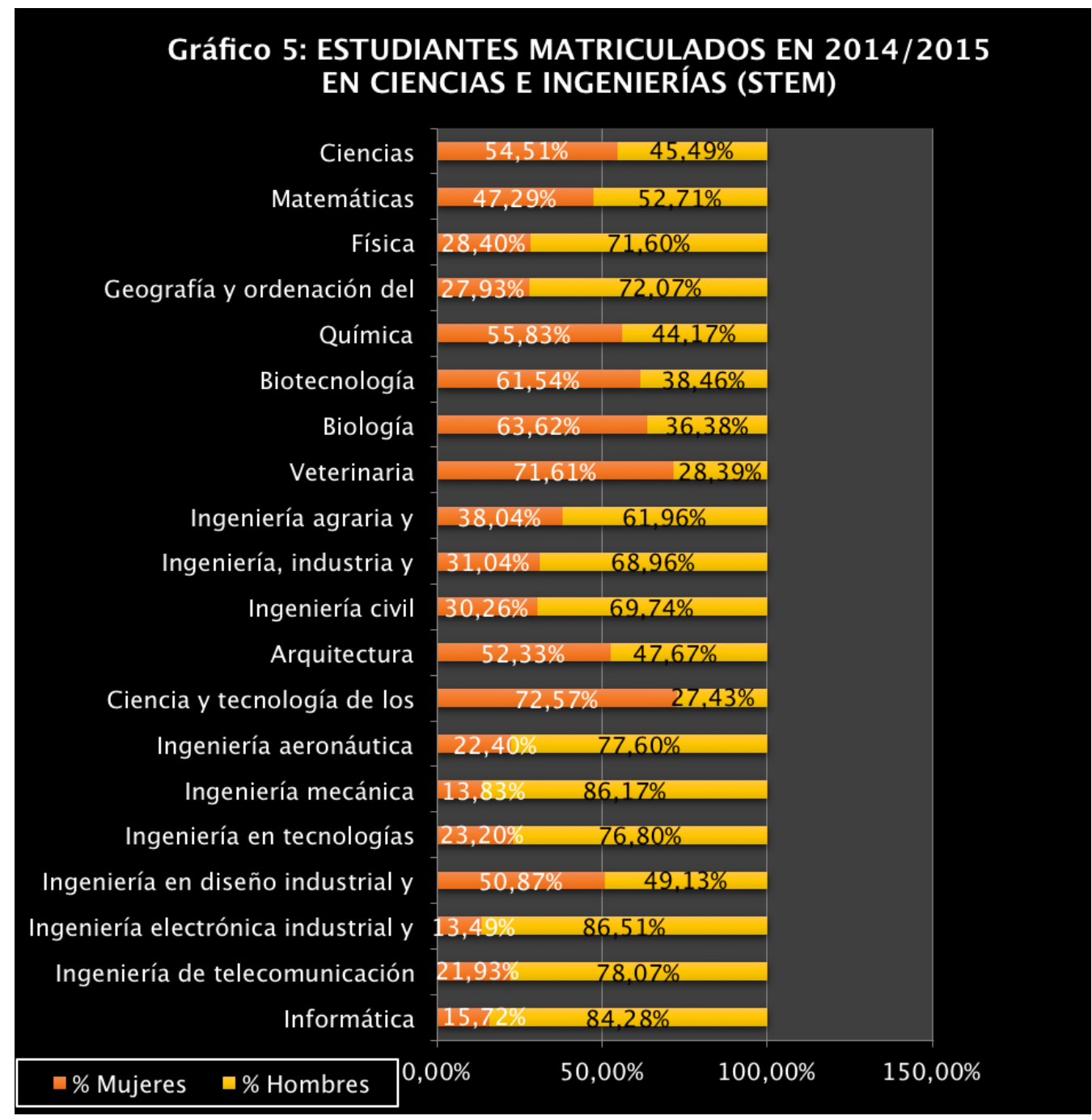

Fuente: Elaboración propia sobre datos S. G. de coordinación y seguimiento universitario. Ministerio de Educación, Cultura y Deporte

de estudiantes de ingeniería informática no se evidenció un problema de renta. En su decisión pesó más contar con referentes cercanos en las carreras de elección. En este sentido, la mitad del grupo tenía padres informáticos o algún ingeniero en la familia, lo que sin duda contribuyó en la reproducción de vocaciones en estas carreras.

Un informe de la UNESCO revela que solo hay un $28 \%$ de científicas en todo el mundo. La infrarrepresentación de mujeres en las STEM es un problema profundamen- 
te arraigado y frena perjudicialmente el progreso hacia el desarrollo sostenible de nuestras sociedades. El déficit de talento femenino forma parte integral de la Agenda 2030 para el Desarrollo Sostenible ${ }^{14}$. Los sistemas educativos y especialmente la escuela juegan un papel determinante en orientar o despertar interés en las niñas por los aspectos positivos de las STEM. Este informe coincide con el informe de la AMIT en señalar que las diferencias de género son visibles en la infancia, y a medida que las niñas avanzan en los cursos, y con la edad, pierden interés por las STEM.

Asimismo, la pedagogía tiene un papel protagonista en el cambio de paradigma educativo en la sociedad del conocimiento o digital. Entre otros muchos pedagogos que están debatiendo sobre la imperante necesidad del cambio metodológico en la educación del siglo XxI, la estadounidense ${ }^{15}$ Davidson sugiere que, a pesar de los cambios en los hábitos de vida y comunicación que ha generado internet, los individuos —en las esferas educativa y laboral - continúan ciegos ante los cambios trascendentales y siguen operando como en el siglo xx. Los modelos de enseñanza y evaluación que sirvieron en la era industrial ya no sirven a una generación de estudiantes que necesitan desarrollar un pensamiento crítico para asimilar y analizar la sobrecarga de información (Davidson, 2011).

El debate sobre las formas de aprendizaje no es nuevo, ya fueron evidenciadas por Gorz durante la primera revolución tecnológica y antes de la irrupción de internet. Gorz decía que los métodos de formación estaban poco adaptados y eran poco motivadores, y animó a realizar una urgente reforma pedagógica en todos los niveles de enseñanza y a poner el acento en el pensamiento crítico y en el desarrollo de competencias que permitieran el acceso a diversos oficios o profesiones. La educación tenía que ayudar a los alumnos a desarrollar capacidades singulares en el ser humano, como plantear preguntas imprevistas, la creatividad y el ingenio (Gorz, 1991:303).

\section{ALGUNAS CONCLUSIONES}

A nadie puede escapársele a estas alturas la evolución de las TIC y su impacto en nuestro estilo de vida, en nuestra forma de comunicarnos y de relacionarnos. Si bien es

${ }^{14}$ Informe UNESCO (2017), Agenda 2030 fue adoptada en 2015 por la Asamblea General de Naciones Unidas.

${ }^{15}$ Cathy Davidson, pedagoga estadounidense y cofundadora de HASTAC (Alianza de humanidades, artes, ciencia, tecnología y colaboración), una red internacional con más de 15000 académicos, artistas y tecnólogos que comparten prácticas en el campo de la enseñanza y el aprendizaje. 
cierto que ya no se puede hablar de sociedad digital como algo que está por venir, creo que tampoco debemos dejarnos llevar por la euforia con la que algunos medios presentan los cambios en las profesiones del futuro. Parte del problema viene de la globalización de la comunicación, que trata las noticias como si todos viviéramos en la misma aldea.

A la luz de los datos empíricos sobre las cifras de matriculación en las carreras tradicionales y las de distribución de profesionales por sexo y sector de actividad, se ha visto que la división del trabajo sigue influenciada de forma significativa por los roles de género aprendidos durante el proceso de socialización desde la infancia hasta el momento de elección de carrera o profesión. En relación con la elección de carrera y la brecha de género en matriculaciones STEM no existen grandes diferencias entre España y los países miembros de la UE. Este dato en sí no sería tan relevante para las autoridades si no fuera por la visibilidad que se le está dando al problema de la escasez de profesionales cualificados en las TIC en detrimento del desarrollo de la economía digital.

También hemos visto que la digitalización de la educación requiere un cambio de paradigma pedagógico que incluya nuevas metodologías de estudio en el sistema educativo, nuevas formas de aprender, repensar y eliminar el aprendizaje memorístico de datos irrelevantes que pueden consultarse de forma instantánea en los diversos dispositivos informáticos que nos acompañan durante las veinticuatro horas. Otra cosa es el mantenimiento del aprendizaje de conocimientos básicos como matemáticas, lengua, física o incluso filosofía, en contra de algunas corrientes de opinión que creen que también pueden eliminarse porque todo se encuentra en internet. Estos conocimientos son vitales para entender el funcionamiento del mundo o para saber de dónde venimos y, en definitiva, para poder discernir entre una información veraz de otra falaz.

El análisis del discurso de los informes presentados en este artículo sugiere al menos dos relatos ocultos relacionados con el déficit vocacional TIC de las mujeres. El primero es sobre la posible exclusión de la mujer en las profesiones del futuro, donde las tecnologías van a ser transversales en todos los sectores de actividad. Como advierten los distintos informes de los organismos consultados, si no se incrementa su presencia, quedarán también excluidas de los centros de poder y decisión en la sociedad digital en ciernes. El segundo relato tiene que ver con la competitividad entre Occidente y las nuevas economías emergentes en China e India, donde sí se está incrementando el número de niñas que eligen estudiar ingenierías TIC. Un hecho que no es ignorado por los gobiernos occidentales, que son conscientes de que la competencia en la economía digital también se mide por el número de efectivos. Precisamente, los países orientales no tienen el problema del envejecimiento en sus sociedades: cuentan con una población mucho más joven, por lo que en pocos años más que déficit de talento digital podrían 
alcanzar un superávit. Esta es una variable de análisis interesante y que he incorporado a mi investigación porque creo que también ha sido una de las razones por las que se está llamando de forma insistente a las mujeres de Occidente al estudio de ingenierías TIC.

\section{REFERENCIAS BIBLIOGRÁFICAS}

Aguinaga, Josune y Comas, Domingo (2013). Informe España 2013, una interpretación de su realidad social. «Capítulo 2: Los jóvenes hoy: aprender a tomar decisiones en un entorno enmarañado». Madrid. Fundación Encuentro.

Aguinaga, Josune (2016). «El uso de las TIC. Su influencia en los cambios individuales y sociales». Revista de Estudios de Juventud. N. ${ }^{\circ} 111: 9-25$. Madrid.

Almerich, G. et al. (2010). «Perfiles del profesorado a partir del conocimiento de los recursos tecnológicos y su relación con el uso que hacen de las tecnologías». Revista Complutense de Educación. Vol. 2 Núm. 2: 247-269. Madrid.

Bauman, Zygmunt (2003). Modernidad líquida. Buenos Aires. Fondo de Cultura Económica de Argentina.

Bourdieu, Pierre (2000). La dominación masculina. Barcelona. Anagrama.

Castaño, Cecilia (2010). Género y TIC: presencia, posición y políticas. Barcelona. $U O C$.

Castaño, Cecilia (2008). La Segunda Brecha Digital. Madrid. Editorial Cátedra.

Castells, Manuel; Tubella, Inma; Sancho, Teresa; Meritxell Roca, (2007). La transición a la sociedad red, Barcelona, Ariel.

Castells, Manuel (2009). Comunicación y poder. Madrid. Alianza Editorial.

Connell, Robert (1987). Gender and Power, Society, the Person and Sexual Politics, Oxford, Polity Press.

Connell, R. W. (2003). Masculinidades, Universidad Nacional Autónoma de México.

Gill, Rosalind (2002). Cool, creative and egalitarian?: exploring gender in project-based new media work in Europe [online]. London: LSE Research Online.

Gorz, Andre (1991). Metamorfosis del trabajo. Búsqueda del sentido. Crítica de la razón económica. Madrid. Editorial Sistema.

Jaúregui, F. et al. (2016). Educa 2020: Universidad y Empleo, manual de instrucciones. Editorial Almuzara.

Leadbeater, C. y Oakley, K. (1999). The Independents. Britain's new cultural entrepreneur. Demos. London.

Moreno, C. (2005). Internet y las competencias básicas: aprender a cooperar, a comunicarse, a participar, a aprender. Editorial Grao. Barcelona. 
Parsons, Talcott (1982). El Sistema Social, 1. a edición, Madrid, Alianza Universidad.

Tezanos, José Félix, (2009). La sociedad dividida. Estructuras de clases y desigualdades en las sociedades tecnológicas, Madrid, Biblioteca Nueva.

\section{Webgrafía}

http://www.minetur.gob.es/es-ES/GabinetePrensa/NotasPrensa/2013/Paginas/npagendadigital150213.aspx (Consultado el 30/06/2017)

Agencia Digital Europea en: http://ec.europa.eu/digital-agenda/ (Consultado el 30/06/2017)

http://www.expansion.com/emprendedores-empleo/empleo/2017/07/10/5963b440ca4741302c8b4636.html (Consultado el 11/07/017)

http://www.industriaconectada40.gob.es/Paginas/Index.aspx\#industria-4 (Consultado el 11/07/2017)

https://educationfutures.com/john/ (Consultado el 11/07/2017)

http://www.sinpermiso.info/textos/taylorismo-digital-la-educacin-no-impedir-que-oc-

cidente-se-empobrezca, (Consultado el 12/07/2017)

https://economia.elpais.com/economia/2016/10/23/actualidad/1477251453_527153.html?id_externo_rsoc=FB_CM (Consultado el 10/08/2017)

https://elpais.com/elpais/2017/08/15/fotorrelato/1502792970_218899.html (Consulta-

do el 16/08/2017) https://elpais.com/elpais/2017/04/07/ciencia/1491558630_

638114.html (Consultado el 16/08/2017)

https://politica.elpais.com/politica/2017/07/07/actualidad/1499459107_578652.ht-

ml?rel=mas (Consultado el 16/08/2017)

https://politica.elpais.com/politica/2017/08/15/actualidad/1502820033_014933.html?id_externo_rsoc=LK_CM

https://info.steelcase.com/global-employee-engagement-workplace-report\#engagement

(Consultado el 10/09/2017)

https://www.mecd.gob.es/servicios-al-ciudadano-mecd/dms/mecd/servicios-al-ciuda-

dano-mecd/estadisticas/educacion/indicadores-publicaciones-sintesis/datos-cifras/

Datosycifras1617esp.pdf (consultado el 21/08/2017)

https:/www.theatlantic.com/technology/archive/2011/08/project-classroom-transforming-our-schools-for-the-future/244182/ (consultado el 22/08/2017)

http://www.informeticplus.com/informe-anual-del-sector-tic-y-de-los-contenidos-enespana-2016-ontsi (Consultado el 10/09/2017) 
https://d2ufo471rtsv5s.cloudfront.net/content/early/2017/04/25/science.aan3264.full (Consultado el 10/09/2017)

https://blogthinkbig.com/menos-del-20-de-las-empresas-en-espana-estan-preparadas-para-la-transformacion-digital (Consultado el 17/09/17)

https:/ec.europa.eu/digital-single-market/digital-economy-and-society-index-desi (Consultado el 17/09/17)

http://www.juntadeandalucia.es/export/drupaljda/Informe_Mujer_y_tecnologia_pdf. pdf (Consultado el 17/09/17)

https://www.mecd.gob.es/servicios-al-ciudadano-mecd/estadisticas/educacion/no-uni-

versitaria/centros/sociedad-informacion/2014-2015.html (Consultado el 30/09/2017)

http://blog.educalab.es/intef/wp-content/uploads/sites/4/2016/03/Resumen_Horizon_

Universidad_2016_INTEF_mayo_2016.pdf (Consultado el 30/09/2017)

http://eur-lex.europa.eu/legal-content/ES/TXT/?uri=LEGISSUM:c11090 (Consultado el 30/09/2017)

http://ec.europa.eu/transparency/regdoc/rep/1/2016/ES/COM-2016-729-F1-ES-MAIN.

PDF (Consultado el 13/10/2017)

http://www.empleo.gob.es/es/sec_trabajo/analisis-mercado-trabajo/pnr/archivos/semestre_axos_anteriores/Informe_conjunto_de_empleo_2015-Borrador-ES.pdf (Consultado el 13/10/2017)

https://elpais.com/economia/2016/12/19/actualidad/1482148215_928653.html (Consultado el 13/10/2017)

http://reports.weforum.org/global-competitiveness-index-2017-2018/ (Consultado el 13/10/2017/) 